\title{
Platelet-Derived Growth Factor Receptor-Alpha D842v Mutation in a Spindle Cell Type Gastrointestinal Stromal Tumor*
}

Jenissa Amor Arceño, ${ }^{1}$ Kathleen Chua, ${ }^{2}$ Loraine Kay Cabral, ${ }^{3}$ Arlie Jean Grace Dumasis, ${ }^{4}$

Jose Jasper Andal, ${ }^{1}$ Raymundo Lo, ${ }^{1}$ Glenda Lyn Pua, ${ }^{1}$ Daphne Ang ${ }^{1}$

${ }^{1}$ Institute of Pathology, St. Luke's Medical Center-Quezon City, Philippines

${ }^{2}$ Department of Surgery, Section of Minimally Invasive Surgery, Chinese General Hospital, Philippines

${ }^{3}$ Research and Biotechnology Division, St. Luke's Medical Center-Quezon City, Philippines

${ }^{4}$ Institute of Pathology, St. Luke's Medical Center-Global City, Philippines

\section{ABSTRACT}

Molecular genotyping of gastrointestinal stromal tumors is not yet available in the Philippines. We report a case of a 75-year old male with a gastric submucosal mass, who underwent gastroscopic/laparoscopic wedge resection. Histopathology and subsequent immunohistochemical staining with CDI17 (CKIT) and DOG1 revealed diagnosis of gastrointestinal stromal tumor, spindle cell variant. On genotyping, the tumor harbored PDGFRA D842V mutation, a subtype resistant to Imatinib treatment.

Key words: gastrointestinal stromal tumor (GIST), platelet derived growth factor receptor-alpha (PDGFRA)

ISSN 2507-8364 (Online)

Printed in the Philippines.

Copyright(C) 2018 by the PJP.

Received: 9 December 2017.

Accepted: 7 February 2018.

Published online first: 8 February 2018.

https://doi.org/10.21141/PJP.2018.004

Corresponding author: Daphne C. Ang, MD

E-mail: daphnechuaang@yahoo.com

*This paper has been presented as a poster at the College of American Pathologists' Meeting 2017, Gaylord National Resort and Convention Center, Prince Georges, Maryland, USA.

\section{INTRODUCTION}

Molecular advancements in pathology have established various mutations in c-KIT and platelet-derived growth factor receptoralpha (PDGFRA) genes, which affect the prognosis and therapy of GIST, specifically their response to tyrosine kinase inhibitors (Imatinib mesylate). Although majority of GIST have mutations in c-KIT gene, mutations in PDGFRA are seen in 5 to $7 \%$ of GISTs. ${ }^{1}$

Of the different PDGFR- $\alpha$ exons involved, a point mutation in exon of PDGFRA D842V has been reported to be notoriously resistant to Imatinib. ${ }^{2}$ In this case report, a mutational analysis is performed on a diagnosed GIST and the presence of a PDGFRA D842V mutant is identified. The importance of molecular subtyping of GISTs, as recommended by the National Comprehensive Cancer Network (NCCN) and European Society for Medical Oncology (ESMO), will also be discussed.

\section{CASE}

A 75-year-old male complained of 6-month history of epigastric pain accompanied by generalized body weakness, early satiety and vomiting of previously ingested food. Endoscopy was done which showed a submucosal mass along the anterior body of the stomach. A subsequent whole abdominal CT scan with contrast was done which showed a soft tissue mass along the anterior mid-body of the stomach, measuring $3 \times 2.4 \mathrm{~cm}$. The patient underwent laparoscopic wedge resection of gastric mass with intraoperative gastroscopy/laparoscopic and endoscopic cooperative surgery (LECS) and the specimen was sent for histopathologic examination (Figure 1).

\section{Histopathology}

Grossly, a light brown to brown, soft to rubbery, irregular tissue fragment was noted measuring 4.8 x $3.5 \times 2.3 \mathrm{~cm}$. Sectioning revealed a $2.5 \times 2.3 \times 2.1 \mathrm{~cm}$. light brown, well- circumscribed mass underneath the mucosa. It has focal areas of hemorrhage. 


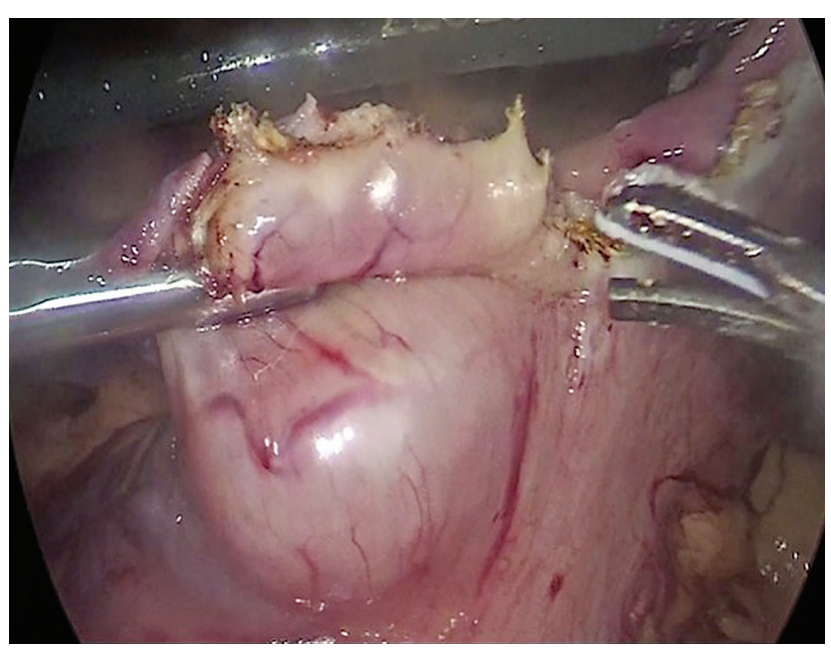

Figure 1. A soft tissue mass along the anterior mid-body of the stomach, measuring $3 \times 2.4 \mathrm{~cm}$.
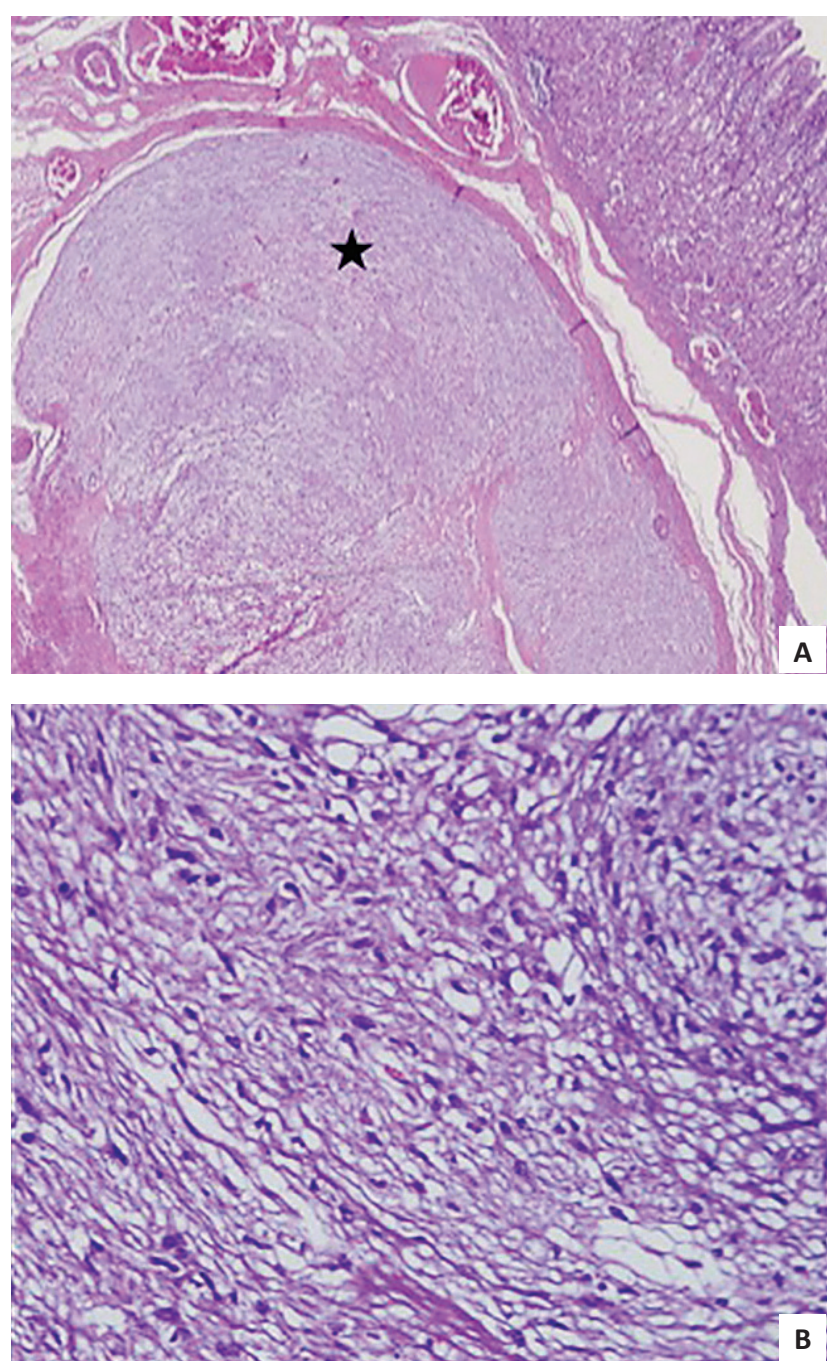

Figure 2. Microscopic sections of gastric mass. (A) Gastric mass at 10X magnification. (B) Gastric mass at 40X magnification.

Microscopy showed nests and sheets of spindle cells infiltrating the muscularis propria. The cells have elongated nuclei, with fine chromatin, inconspicuous nuclei and moderate amount of pale eosinophilic and fibrillary cytoplasm (Figure 2). Mitotic count

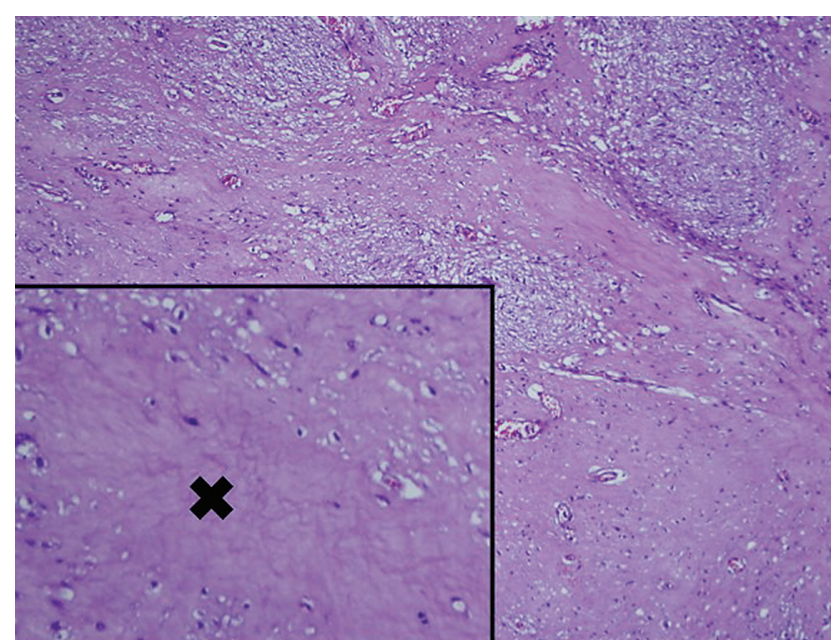

Figure 3. Gastric mass with areas of necrosis $(\mathbf{X})$.

was 0 to 1 per 50 high power fields. However, areas of necrosis were seen in the specimen (Figure 3). The immunohistochemical stains done showed diffusely positive CD117 and DOG1, and negative for Desmin and S100, supporting the diagnosis of gastrointestinal stromal tumor (Figure 4). Based on the National Comprehensive Cancer Network (NCGN), it was stratified as having very low risk for progressive disease.

\section{Molecular Testing}

The tumor block was submitted for mutational analysis by Next Generation Sequencing carried out using Illumina MiSeq (San Diego, California). Briefly, the TruSight Tumor 15 (TST) assay panel screens for hotspot regions in 15 genes (AKT1, BRAF, EGFR, ERBB2, FOXL2, GNA11, GNAQ, KIT, KRAS, MET, NRAS, PDGFRA, PICK2CA, RET and TP53), across 250 amplicons.

DNA was extracted from $6 \times 4 \mathrm{~mm}$ sections of the tumor block using Qiagen FFPE kit and DNA was quantitated using qubit. An extension and ligation-based amplification library preparation assay specific for each of the two strands of DNA was used for all targets and the index sequence was incorporated into tailed universal PCR primers. The resulting libraries were then sequenced using Illumina MiSeq with a minimum read depth of at least 1000x coverage for all amplicons. Demultiplexing and read alignment was then performed using the reference genome UCSG HG19 human build. The Illumina somatic variant caller was used for variant calling.

Sanger sequencing was performed to confirm the positive result (Figure 5). PDGFR- $\alpha$ exon 18 was amplified using the following primers: forward primer 5'CAGTACACAGATGGCTTGATC3' and reverse primer 3'TGAAGGAGGATGAGGCTGAC5'.

\section{DISCUSSION}

Gastrointestinal stromal tumors are mesenchymal tumors commonly arising in the stomach $(60 \%)$, followed by jejunum and ileum $(30 \%)$, duodenum $(5 \%)$ and colorectal $(<5 \%) .{ }^{3}$ In the absence of mutational testing, diagnosis of GIST relies heavily on immunohistochemical staining with CD117 and DOG1, of which the patient tested positive. These immunohistochemical stains can detect most GISTs except for a few $(3 \%$ to $5 \%)$ that harbor PDGFRA mutation. ${ }^{4}$ 

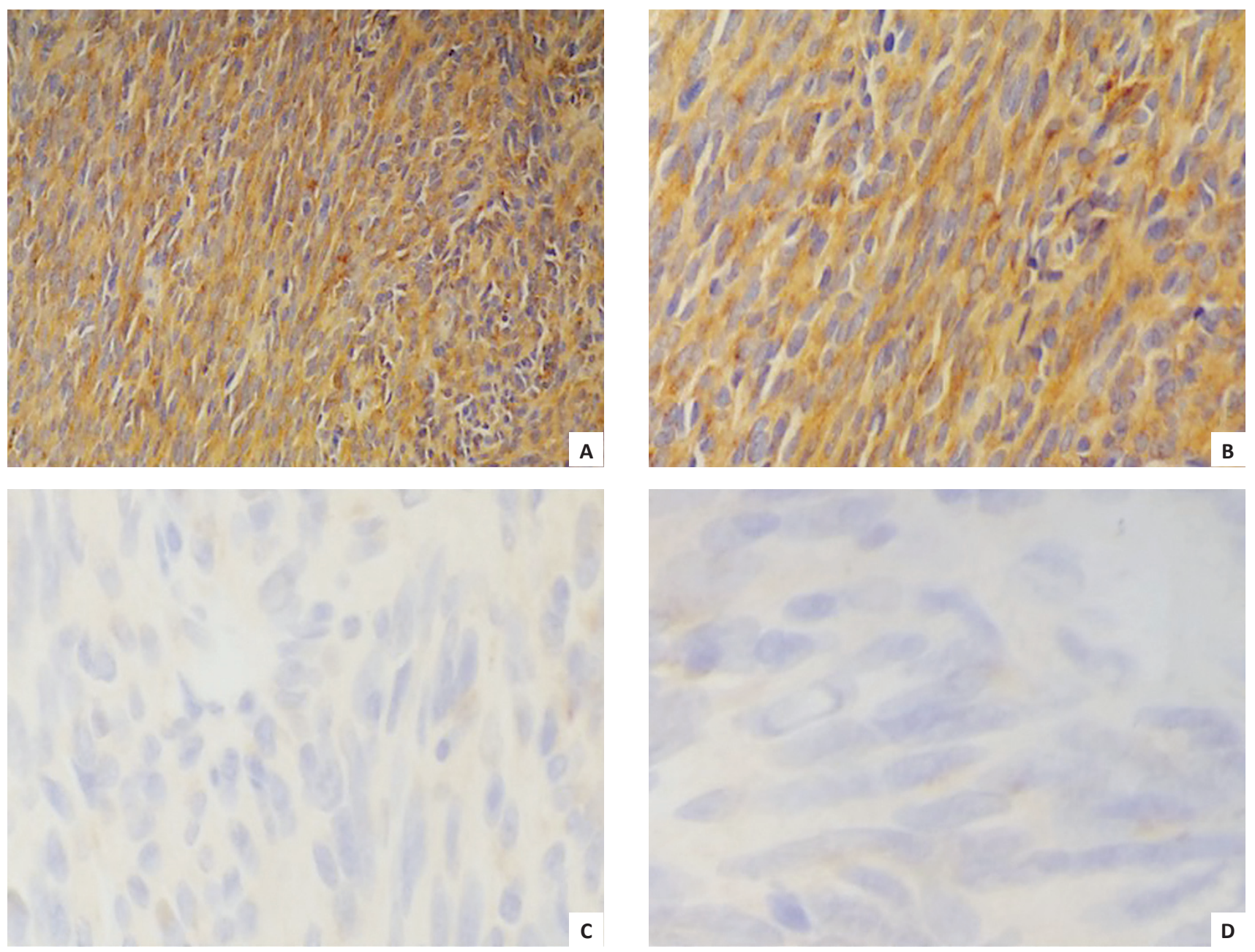

Figure 4. Immunohistochemical stains of the gastric mass. The tumor cells are positive in (A) DOG1 and (B) CD117; and negative in (C) S100 and (D) Desmin.

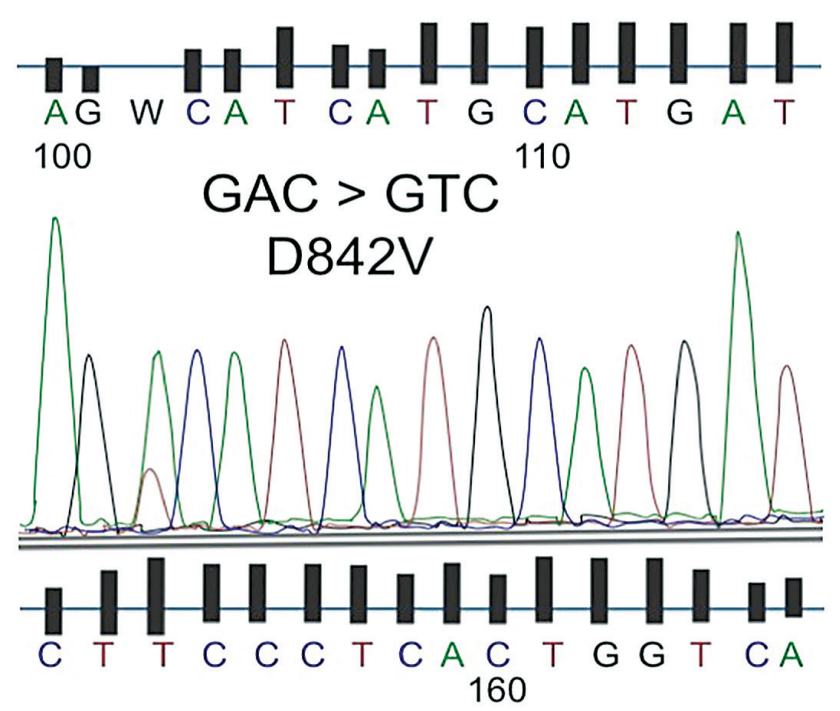

Figure 5. Sanger sequencing result of PDGFRA exon 18 showing D842V mutation.

Gastrointestinal stromal tumors have activating kit (CD117)positive or platelet-derived growth factor receptor alpha (PDGFRA) mutations resulting in the constitutive activation of protein tyrosine kinase signaling. ${ }^{5-7}$ The most common
KIT mutations are detected in exon $11(66-71 \%)$, exon 9 (10$13 \%)$, exon $13,14,17(1 \%$ each). PDGFRA mutations $(8 \%)$ are described in exon $18(5-6 \%)$, exon $12(1 \%)$ and exon $14(1 \%){ }^{4}$ Of importance in this case is the PDGFRA mutation at exon 18, with the missense mutation leading to an amino acid change from aspartic acid to valine (D842V). Tumors with PDGFRA D842V usually have an epithelioid morphology, indolent course and remain localized with low risk of recurrence. ${ }^{8-9}$

However, GISTs harboring this mutation are usually resistant to Imatinib, ${ }^{1}$ Interestingly, our case, although PDGFRA positive, histomorphology exhibited spindle cell features. While the tumor size and mitotic count favor a very low risk of progression, the presence of tumor necrosis may indicate a worrisome feature that may warrant close follow up. In a study done by Liang et al, 2007 , included in the clinicopathologic parameters that indicate worse prognosis are advanced clinical stage, tumor diameter, mitotic index, coagulative necrosis and risk grade. ${ }^{10}$

Some reports show aggressive behavior of PDGFRA mutants. ${ }^{11}$ It is believed that the resistance of PDGFRA-mutant GISTs correlates selectively with the substitution mutations that affect codon D842V of the activation loop, which is associated with a conformational shift of the adenosine triphosphate (ATP)binding pocket from an "open" or active conformation to a "closed" or inactive conformation. ${ }^{12}$ 
In a study of 18 participants with PDGFRA mutation who were treated with first line Imatinib, the authors found a significantly different objective response rate between patients with the D842V mutation and those with non-D842V mutations $(0 \%$ [0/5] vs. $71 \%$ [5/7], $\mathrm{p}=0.03)$. They also found a significantly poorer progression free survival between D842V mutations than those with non-D $842 \mathrm{~V}$ mutant GISTs: median 3.8 months vs. 29.5 months $(\mathrm{p}<0.001) .^{13}$

\section{CONCLUSION}

This case of PDGFRA D842V mutant GIST, histologically classified as spindle cell type with very low risk of progressive disease, raises possibilities that spindle cell type GISTs resistant to standard treatment are present within our population. Hence, mutational profiling of gastrointestinal stromal tumors should be incorporated to routine clinical practice. These patients should be encouraged to participate in clinical trials for investigational drugs with potential activity against this mutation. ${ }^{12}$

For this case, surgery still remains as the primary mode of treatment. ${ }^{11}$ With the negative surgical margins, tumor size of $2.5 \mathrm{~cm}$. and mitotic count of 0 to 1 per 50 high power fields, NCGN recommends observation and surveillance. To address this, the patient should be followed up every 3 to 6 months, with recommendations of imaging modalities.

\section{ETHICAL CONSIDERATION}

All efforts to secure patient's consent have been exhausted. The patient's anonymity is ensured. No other identifiers were included.

\section{STATEMENT OF AUTHORSHIP}

All authors certified fulfillment of ICMJE authorship criteria.

\section{AUTHOR DISCLOSURE}

The authors declared no conflict of interest.

\section{FUNDING SOURCE}

None.

\section{REFERENCES}

1. Miettinen M, Lasota J. Gastrointestinal stromal tumors: review on morphology, molecular pathology, prognosis, and differential diagnosis. Arch Path Lab Med. 2006; 130(10)1466-78. PMID: 17090188.

2. Pogorzelski M, Falkenhorst J, Bauer S. Molecular subtypes of gastrointestinal stromal tumor requiring specific treatments. Curr Opin Oncol. 2016;28(4):331-7. PMID: 27163723. https://doi.org/10.1097/CCO.0000000000000303.
3. Xu Z, Huo X, Tang C, et al. Frequent KIT mutations in human gastrointestinal stromal tumors. Sci Rep. 2014;4:5907. PMID: 25080996. PMCID: PMC4118194. https://doi.org/10.1038/srep05907.

4. Comandone A, Boglione A. The importance of mutational status in prognosis and therapy of GIST. Recenti Prog Med. 2015;106(1):17-22. PMID: 25621775. https://doi. org/10.1701/1740.18950.

5. Heinrich MC, Corless CL, Demetri GD, et al. Kinase mutations and imatinib response in patients with metastatic gastrointestinal stromal tumor. J Clin Oncol. 2003;21(23):4342-9. PMID: 14645423. https://doi.org/ 10.1200/JCO.2003.04.190.

6. Nilsson B, Bumming P, Meis-Kindblom JM, et al. Gastrointestinal stromal tumor: the incidence, prevalence, clinical course and prognostication in the preimatinib mesylate ear - a population-based study in western Sweden. Cancer. 2005;103(4):821-9. PMID: 15648083. https://doi. org/10.1002/cncr.20862.

7. Lasota J, Miettinen M. Clinical significance of oncogenic KIT and PDGFRA mutations in gastrointestinal stromal tumours. Histopathology. 2008;53(3):245-66. PMID: 18312355. https://doi.org/10.1111/j.1365-2559.2008.02977.x.

8. Emile JF, Brahimi S, Coindre JM, et al. Frequencies of KIT and PDGFRA mutations in the MolecGIST prospective population-based study differ from those of advanced GISTs. Med Oncol. 2012;29(3):1765-72. PMID: 21953054. https://doi.org/10.1007/s12032-011-0074-y.

9. Cassier PA, Ducimetière F, Lurkin A, et al. A prospective epidemiological study of new incident GISTs during two consecutive years in Rhone Alpes region: incidence and molecular distribution of GIST in a European region. $\mathrm{Br}$ J Cancer. 2010; 103(2):165-70. PMCID: PMC2906738. https://doi.org/10.1038/sj.bjc.6605743.

10. Liang YM, Li XH, Lü YY, Lü YL, Zhong M, Pu XL, Li WM. Prognostic significance of clinicopathologic parameters in gastrointestinal stromal tumor: a study of 156 cases. Zhonghua Bing Li Xue Za Zhi. 2007;36(4):233-8. PMID: 17706113 .

11. Tan CB, Zhi W, Shahzad G, Mustacchia P. Gastrointestinal stromal tumors: a review of case reports, diagnosis, treatment and future directions. ISRN Gastroenterol. 2012 2012: 595968. PMID: 22577569. PMCID: PMC3332214. https://doi.org/10.5402/2012/595968.

12. Corless CL, Shroeder A, Griffith D, et al. PDGFRA mutations in GISTs: frequency, spectrum and in vitro sensitivity to imatinib. J Clin Oncol. 2005;23(23):5357-64. PMID: 15928335. https://doi.org/10.1200/JCO.2005.14.068.

13. Yoo C, Ryu M, et al. Efficacy of imatinib in patients with platelet-derived growth factor receptor alpha-mutated gastrointestinal stromal tumors. Cancer Res Treat. 2016;48(2):546-52. PMCID: PMC4843750. https://doi. org/10.4143/crt.2015.015.

Disclaimer: This journal is OPEN ACCESS, providing immediate access to its content on the principle that making research freely available to the public supports a greater global exchange of knowledge. As a requirement for submission to the PJP, all authors have accomplished an AUTHOR FORM, which declares that the ICMJE criteria for authorship have been met by each author listed, that the article represents original material, has not been published, accepted for publication in other journals, or concurrently submitted to other journals, and that all funding and conflicts of interest have been declared. Consent forms have been secured for the publication of information about patients or cases; otherwise, authors have declared that all means have been exhausted for securing consent. 